\title{
Epigenome-wide association study of Alzheimer's disease replicates 22 differentially methylated positions and 30 differentially methylated regions
}

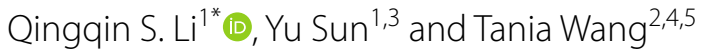

\begin{abstract}
Background: Growing evidence shows that epigenetic modifications play a role in Alzheimer's disease (AD). We performed an epigenome-wide association study (EWAS) to evaluate the DNA methylation differences using postmortem superior temporal gyrus (STG) and inferior frontal gyrus (IFG) samples.

Results: Samples from 72 AD patients and 62 age-matched cognitively normal controls were assayed using Illumina ${ }^{\circledR}$ Infinium MethylationEPIC BeadChip. Five and 14 differentially methylated positions (DMPs) associated with pathology (i.e., Braak stage) with p value less than Bonferroni correction threshold of $6.79 \times 10^{-8}$ in the STG and IFG were identified, respectively. These cytosine-phosphate-guanine $(\mathrm{CpG})$ sites included promoter associated cg26263477 annotated to ABCA7 in the STG $\left(p=1.21 \times 10^{-11}\right)$, and cg14058329 annotated to the HOXA5/HOXA3/HOXA-AS3 gene cluster $\left(p=1.62 \times 10^{-9}\right)$ and $c g 09448088\left(p=3.95 \times 10^{-9}\right)$ annotated to MCF2L in the IFG. These genes were previously reported to harbor DMPs and/or differentially methylated regions (DMRs). Previously reported DMPs annotated to RMGA, GNG7, HOXA3, GPR56, SPG7, PCNT, RP11-961A15.1, MCF2L, RHBDF2, ANK1, PCNT, TPRG1, and RASGEF1C were replicated $(p<0.0001)$. One hundred twenty-one and 173 DMRs associated with pathology in the STG and IFG, respectively, were additionally identified. Of these, DMRs annotated to 30 unique genes were also identified as significant DMRs in the same brain region in a recent meta-analysis, while additional DMRs annotated to 12 genes were reported as DMRs in a different brain region or in a cross-cortex meta-analysis. The significant DMRs were enriched in promoters, CpG islands, and exons in the genome. Gene set enrichment analysis of DMPs and DMRs showed that gene sets involved in neuroinflammation (e.g., microglia differentiation), neurogenesis, and cognition were enriched (false discovery rate $(F D R)<0.05)$.
\end{abstract}

Conclusions: Twenty-two DMPs and 30 DMRs associated with pathology were replicated, and novel DMPs and DMRs were discovered.

Keywords: Epigenetics, EWAS, DMP, DMR

*Correspondence: qli2@its.jnj.com

${ }^{1}$ Neuroscience, Janssen Research \& Development, LLC, 1125 Trenton-Harbourton Road, Titusville, NJ 08560, USA

Full list of author information is available at the end of the article

\begin{abstract}
Introduction
Dementia refers to conditions of memory loss and other cognitive decline serious enough to interfere with daily life. Alzheimer's disease (AD) is the most common cause of dementia and accounts for $50-75 \%$ of dementia cases [1]. While genetic studies have identified familial risk factors such as APP, PSEN1, and PSEN2 that are
\end{abstract} original author(s) and the source, provide a link to the Creative Commons licence, and indicate if changes were made. The images or other third party material in this article are included in the article's Creative Commons licence, unless indicated otherwise in a credit line to the material. If material is not included in the article's Creative Commons licence and your intended use is not permitted by statutory regulation or exceeds the permitted use, you will need to obtain permission directly from the copyright holder. To view a copy of this licence, visit http://creativecommons.org/licenses/by/4.0/. The Creative Commons Public Domain Dedication waiver (http://creativeco mmons.org/publicdomain/zero/1.0/) applies to the data made available in this article, unless otherwise stated in a credit line to the data. 
involved with amyloid- $\beta$ production, they only account for a small fraction of patients with early onset AD [2]. Most patients with $\mathrm{AD}$ acquire the disease late in life (i.e., age of onset $>65$ years). Genome-wide association studies (GWAS) have identified dozens of loci for late onset AD (LOAD) [3-9]. Most of the variants confer risk with relatively small effect size, except for $A P O E$ variants with modest effect size. Next generation sequencing (NGS) enables rare variant analysis to further identify genes such as TREM2 playing a critical role in AD [9-11]. Growing evidence shows that epigenetic modifications also play a role in AD onset and progression [12-15]. Epigenetic modification could be detected by bisulfite conversion, a method that assesses the degree of DNA methylation present as it converts unmethylated cytosine to uracil (and to thymine through PCR), while 5-methylcytosine $(5-\mathrm{mC})$ and 5-hydroxymethylcytosine (5-hmC) are not converted (and thus remain as cytosine in PCR). As such, bisulfite treatment of DNA allows the differentiation between cytosine and the modified versions of cytosine $(5-\mathrm{mC} / 5-\mathrm{hmC})$ through downstream assay techniques. Oxidative bisulfite conversion may further distinguish 5-mC from 5-hmC [16]. Epigenome-wide association studies (EWAS) using bisulfite conversion approaches coupled with the Illumina Infinium ${ }^{\circledR}$ HumanMethylation 450 BeadChip have demonstrated robust and reproducible differences in total DNA methylation at a number of loci in $\mathrm{AD}$ brain [17-22], including ankyrin 1 (ANK1), ABCA7, BIN1, TREM2, and the HOXA and $H O X B$ gene clusters. Notably, a recent meta-analysis using samples from 6 cohorts identified a total of 220 DMPs in a cross-cortex meta-analysis [21].

Volumetric measurements of specific regions of the cortex from AD patients reveal anatomical regions with severe, moderate, or mild/no atrophy. Severe atrophy occurs in medial temporal lobe structures as well as in inferior temporal and superior and middle frontal cortices, while moderate atrophy takes place in the superior temporal gyrus (STG) and no atrophy is noted in the inferior frontal lobes [23]. While there is no significant increase in total (intra- and extracellular) neurofibrillary tangles (NFTs), moderate neuronal loss and evidence of oxidative stress are observed in the STG [24-28]. The STG could therefore be a surrogate for an earlier stage compared to the most severely affected regions, while inferior frontal gyrus (IFG) could represent the earliest stage in the disease course. In this EWAS study, total methylation patterns from the STG and IFG were interrogated using Infinium ${ }^{\circledR}$ MethylationEPIC BeadChip containing $\sim 850 \mathrm{~K} \mathrm{CpG}$ probes, doubling the density of the Infinium ${ }^{\circledR}$ HumanMethylation450 BeadChip used in earlier studies $[17,18,20,29]$. DMPs and DMRs associated with pathology were identified. Additionally, we performed a replication study using this study data set to replicate the findings from a recent EWAS meta-analysis [21]. Gene set enrichment and over representation analyses were performed to provide insight into coherent biological pathways and processes.

\section{Results}

\section{Postmortem brain tissue epigenetic profiling and DMP analysis results}

Demographic and clinical characteristics are provided in Table 1. The mean Pearson correlations of methylation levels for all possible subject pairs were 0.986 and 0.984 for the STG and IFG samples, respectively, indicating that the majority of the CpG sites did not show significant differences in DNA methylation levels. The estimated proportion of $\mathrm{NeuN}^{+}$cells (primarily neurons) showed no significant differences between AD patients and cognitively normal controls (Wilcoxon ranksum test $p$ value $=0.504$ and 0.159 in the STG and IFG, respectively).

Epigenome-wide association studies are known to be prone to significant inflation and bias of test statistics. Lambda $(\lambda)$ inflation factors were 1.54 and 1.11 for the initial EWAS in the STG and IFG, respectively, suggesting the presence of inflation in test statistics (Additional file 1: Figure S1 for QQ plots). A Bayesian method based on estimation of the empirical null distribution as implemented in BACON [30] was used to control bias and inflation in EWAS. After BACON correction, $\lambda$ values for both EWAS were less than 1.05 in this study. All results reported in this study are after BACON correction. Manhattan plots are also available in Additional file 1: Figure S2.

Five CpGs were associated with pathology in the STG passing Bonferroni correction threshold of $6.79 \times 10^{-8}$, including cg26263477 annotated to $A B C A 7$ $\left(p=1.21 \times 10^{-11}\right.$, Table 2$)$, a gene known to harbor an $A D$ susceptibility genetic variant and DMP [5, 31]. In addition, fourteen $\mathrm{CpG}$ probes were associated with pathology in the IFG, including CpG probes cg14058329 annotated to the HOXA5/HOXA3/HOX-AS3 gene cluster $\left(p=1.62 \times 10^{-9}\right)$ and $\operatorname{cg} 09448088\left(p=3.95 \times 10^{-9}\right)$ annotated to $M C F 2 L$ (Fig. 1). cg09448088 was recently reported as a significant DMP in an cross-cortex Braak stage EWAS meta-analysis [21], and the HOXA gene cluster was reported to harbor DMPs and DMRs associated with Braak stage [20,21]. There is no overlap between the study-wide significant findings between these two brain regions.

Of the 14 significant DMPs associated with pathology in the IFG, 5 were nominally associated with pathology $(p<0.05)$ in the STG. The effect sizes in the STG for the 19 DMPs associated with pathology in either the STG 
Table 1 Demographic and clinical characteristics of the samples used in the EPIC array assay

\begin{tabular}{|c|c|c|c|c|c|c|}
\hline \multirow{3}{*}{$\begin{array}{l}\text { Brain region } \\
\text { Clinical diagnosis } \\
\text { Sample size }\end{array}$} & \multicolumn{3}{|l|}{ STG } & \multicolumn{3}{|l|}{ IFG } \\
\hline & Cognitively normal & Alzheimer's disease & $p^{* *}$ & Cognitively normal & Alzheimer's Disease & $p^{* *}$ \\
\hline & $N=60$ & $N=67$ & & $N=57$ & $N=60$ & \\
\hline Age at death (year), mean (SD) & $80.65(6.94)$ & $81.00(7.09)$ & 0.717 & $81.00(6.63)$ & $81.13(6.40)$ & 0.967 \\
\hline Sex, male $n(\%)$ & $37(61.7)$ & $39(58.2)$ & 0.692 & $36(63.2)$ & $35(58.3)$ & 0.593 \\
\hline PMI (hour), Mean (SD) & $3.24(2.02)$ & $3.06(1.60)$ & 0.837 & $3.25(2.05)$ & $3.15(1.69)$ & 0.846 \\
\hline Estimated NeuN ${ }^{+}(\%)$ Mean (SD) & $30.3(13.1)$ & $29.6(11.3)$ & 0.504 & $24.5(12.3)$ & $28.1(9.9)$ & 0.159 \\
\hline Estimated NeuN- (\%), Mean (SD) & $68.6(14.1)$ & $69.3(12.7)$ & 0.570 & $69.0(15.4)$ & $64.1(12.0)$ & 0.095 \\
\hline \multicolumn{7}{|l|}{ NIA-Reagan criteria [72], $n$} \\
\hline Criteria not met & 59 & & & 56 & & \\
\hline Not AD & 1 & & & 1 & & \\
\hline Low & & 2 & & & 2 & \\
\hline Intermediate & & 16 & & & 17 & \\
\hline High & & 49 & & & 41 & \\
\hline \multicolumn{7}{|c|}{ Semiquantitative measure of neuritic plaques CERAD score [73], $n$} \\
\hline Criteria not met & 6 & & & 6 & & \\
\hline Not AD & 33 & & & 31 & & \\
\hline Possible AD & 21 & & & 20 & & \\
\hline Probable AD & & 8 & & & 8 & \\
\hline Definite AD & & 59 & & & 52 & \\
\hline \multicolumn{7}{|l|}{ Braak stage, $n$} \\
\hline I & 15 & & & 12 & & \\
\hline$\|$ & 14 & 2 & & 14 & 2 & \\
\hline III & 20 & 4 & & 21 & 4 & \\
\hline IV & 11 & 12 & & 10 & 13 & \\
\hline V & & 28 & & & 21 & \\
\hline $\mathrm{Vl}$ & & 21 & & & 20 & \\
\hline \multicolumn{7}{|l|}{ APOE genotype, $n^{*}$} \\
\hline$\varepsilon 2 / \varepsilon 2$ & 1 & 0 & & 2 & 0 & \\
\hline$\varepsilon 2 / \varepsilon 3$ & 10 & 3 & & 10 & 1 & \\
\hline$\varepsilon 3 / \varepsilon 3$ & 32 & 26 & & 30 & 26 & \\
\hline$\varepsilon 3 / \varepsilon 4$ & 16 & 33 & & 15 & 27 & \\
\hline$\varepsilon 2 / \varepsilon 4$ & 0 & 1 & & 0 & 1 & \\
\hline$\varepsilon 4 / \varepsilon 4$ & 1 & 3 & & 0 & 4 & \\
\hline
\end{tabular}

SD: standard deviation; STG: superior temporal gyrus (BA22); IFG: inferior frontal gyrus (BA44); PMI: postmortem interval; CERAD [73]: Consortium to Establish a Registry for Alzheimer's Disease

*1 STG and 1 IFG sample, respectively, has missing APOE genotype

**Wilcoxon rank-sum test for continuous variables; Chi-squared test for categorical variables

or IFG were correlated with the effect sizes for the same probes in the IFG $(r=0.50, p=0.03)$ (Fig. 2a). A full list of DMPs associated with pathology with $\mathrm{p}$ value less than $6.79 \times 10^{-8}$ in either the STG or IFG is available in the Additional file 2: Table S1.

Using the reported significant DMP findings from a recent EWAS meta-analysis [21], our results replicated a subset of reported epigenome-wide significant DMPs. There were 377 unique genome-wide significant CpGs (236, 95, 10, and 220 CpGs associated with Braak stage in the prefrontal cortex (PFC), temporal gyrus (TG), EC, and cross-cortex, respectively) reported, among which 344 CpGs passed QC and were present in this dataset. $236(68.6 \%)$ of these 344 CpGs were nominally significant $(p<0.05)$ in our study, while $22(6.4 \%)$ remained significant after accounting for the multiple tests of the replication effort $(p<0.05 / 344 \sim 0.0001)$ (Table 2$)$. The replication rate in the IFG seemed to be higher than that in the STG. Eighteen (8.33\%) out of 216 significant DMPs in the PFC (216 out of 236 DMPs from the meta-analysis 







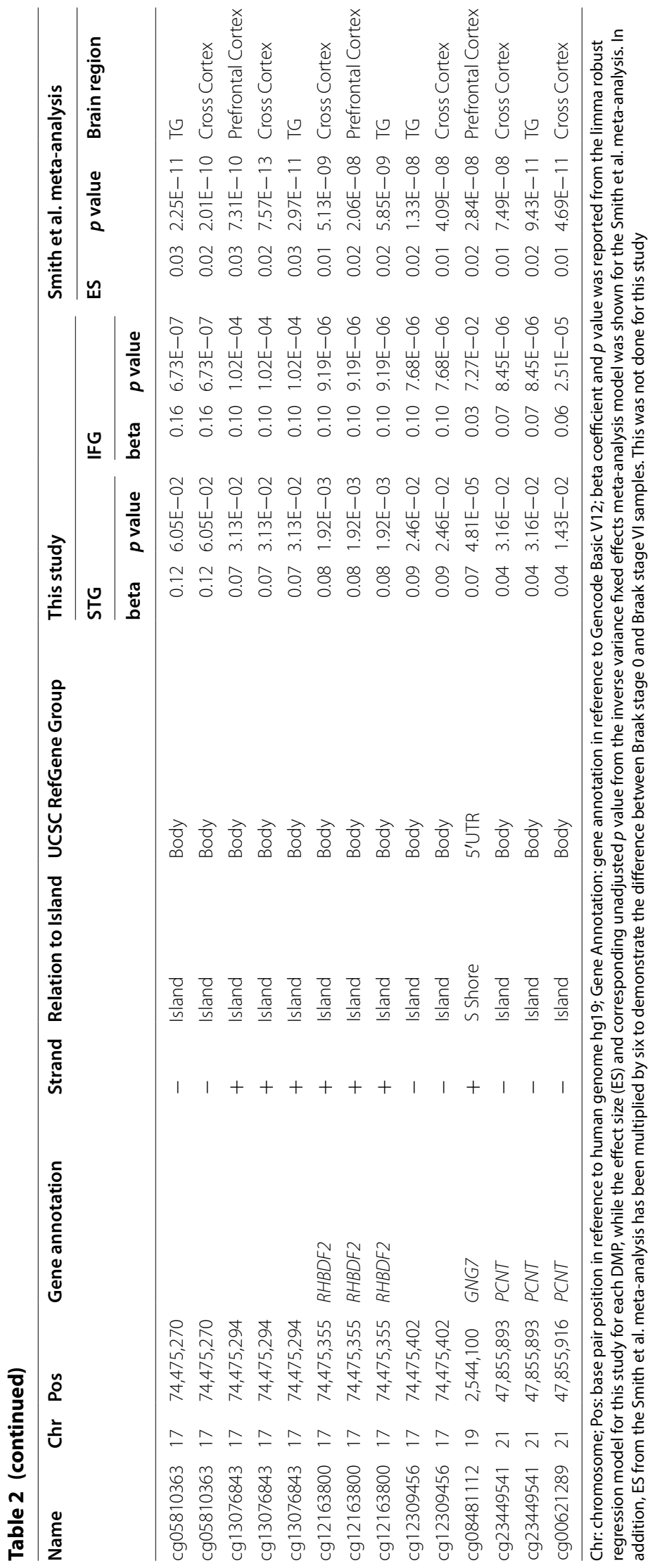


HOXA5 cg14058329

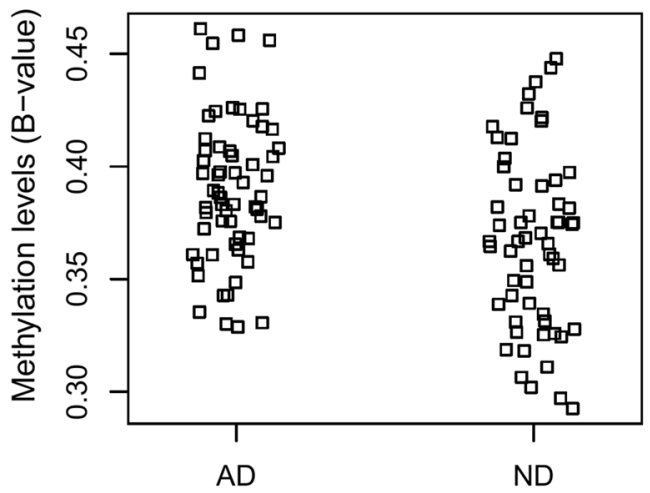

MCF2L cg09448088

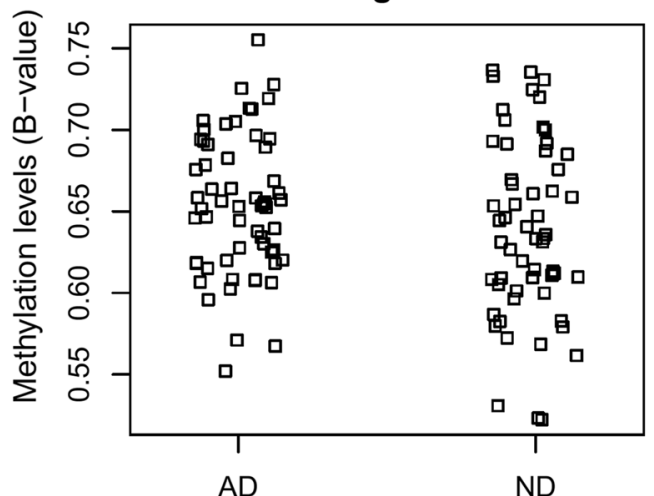

HOXA5 cg14058329

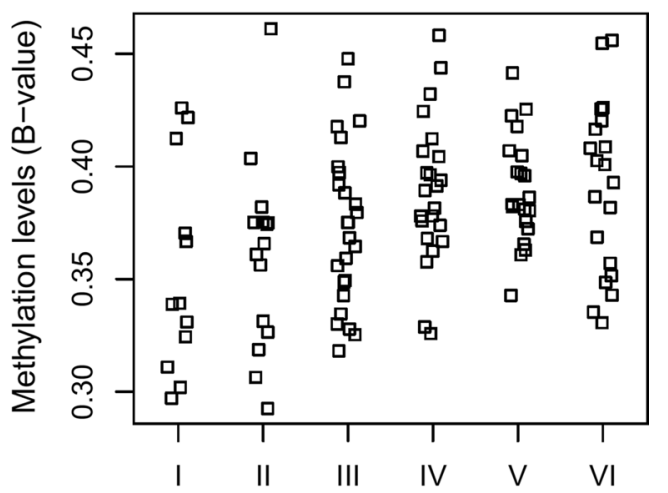

MCF2L cg09448088

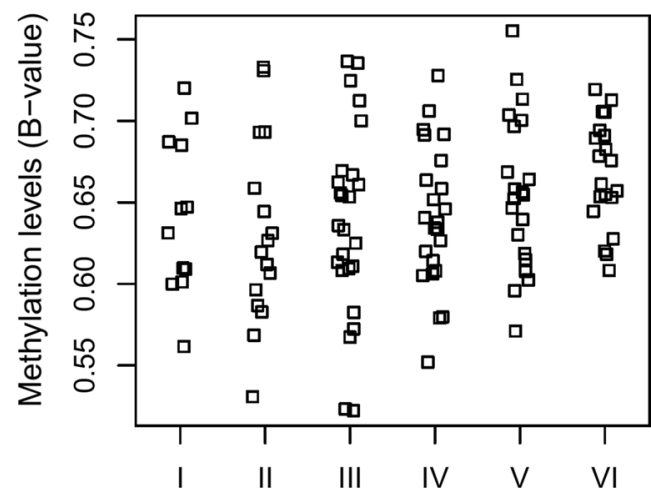

Fig. 1 DMP associations with diagnosis and pathology. The methylation level as measured by B value for probe cg14058329 annotated to HOXA5 was plotted again AD diagnosis (a) and Braak stage (b). Similarly, B value for probe cg09448088 annotated to MCF2L was plotted again diagnosis (c) and Braak stage $(\mathbf{d})$. In both cases, hypermethylation was observed in later Braak stage than earlier stage

passed QC in this study) were replicated in the IFG analysis, as opposed to $4(4.49 \%)$ out of 89 (89 out of 95 significant DMPs from the TG meta-analysis passed QC) were replicated in the STG analysis. The replicated DMPs included probes annotated to genes in RMGA, GNG7, HOXA3, GPR56, SPG7, PCNT, RP11-961A15.1, MCF2L, RHBDF2, ANK1, PCNT, TPRG1, and RASGEF1C. The effect sizes in the STG for the Braak stage association were correlated with those in the meta-analysis except for the EC (Fig. $2 \mathrm{~b}, r=0.77,0.78,0.77$, all $p<2.2 \times 10^{-16}$ for cross-cortex, PFC, TG, respectively; $r=0.32, p$ value $=0.40$ for $E C$ ). The same was true for the effect sizes in the IFG except for the EC when compared to the meta-analysis effect sizes (Fig. $2 c, r=0.78, p<2.2 \times 10^{-16}$ for cross-cortex; $r=0.77, p<2.2 \times 10^{-16}$ for the PFC; $r=0.70, p=3.38 \times 10^{-14}$ for the TG; and $r=0.08, p=0.83$ for the EC).

\section{DMR analysis results}

A DMR analysis, which allowed us to identify regions of the genome consisting of $\geq 3$ probes, revealed a total of 121 and 173 DMRs significantly associated with the pathology in the STG and IFG, respectively (Sidak-corrected $p$ value $<0.05$, Additional file 2 : Table $\mathrm{S} 2 \mathrm{~A}$ and S2B), among which 11 and 33 were reported to be significant DMRs associated with pathology identified in the corresponding cortex region in the recent EWAS metaanalysis [21]. Lists of replicated DMRs in the same brain region and more broadly in any brain region are available in Additional file 2: Table S3A and S3B. The most striking genomic regions associated with pathology in the IFG are 6 DMRs spanning HOXA2/HOXA3/HOXA-AS2/HOXA5 consisting of a total of 79 probes (Additional file 1: Figure S3A). This gene cluster is composed of DMRs in HOXA3 (chr7: 27,153,580-27,155,548 [23 probes], Sidakcorrected $\left.p=1.70 \times 10^{-8}\right)$; HOXA-AS2 (chr7:27,161,749$27,163,095$ [11 probes], Sidak-corrected $p=3.65 \times 10^{-9}$ ), HOXA5 (chr7:27,183,274-27,184,375 [25 probes], Sidakcorrected $\left.p=7.62 \times 10^{-6}\right)$; HOXA2 (chr7:27,143,046$27,143,806$ [11 probes], Sidak-corrected $p=2.65 \times 10^{-7}$; chr7:27,145,972-27,146,445 [5 probes], Sidak-corrected $p=8.86 \times 10^{-5} ; \quad$ and $\operatorname{chr} 7: 27,150,031-27,150,403$ 


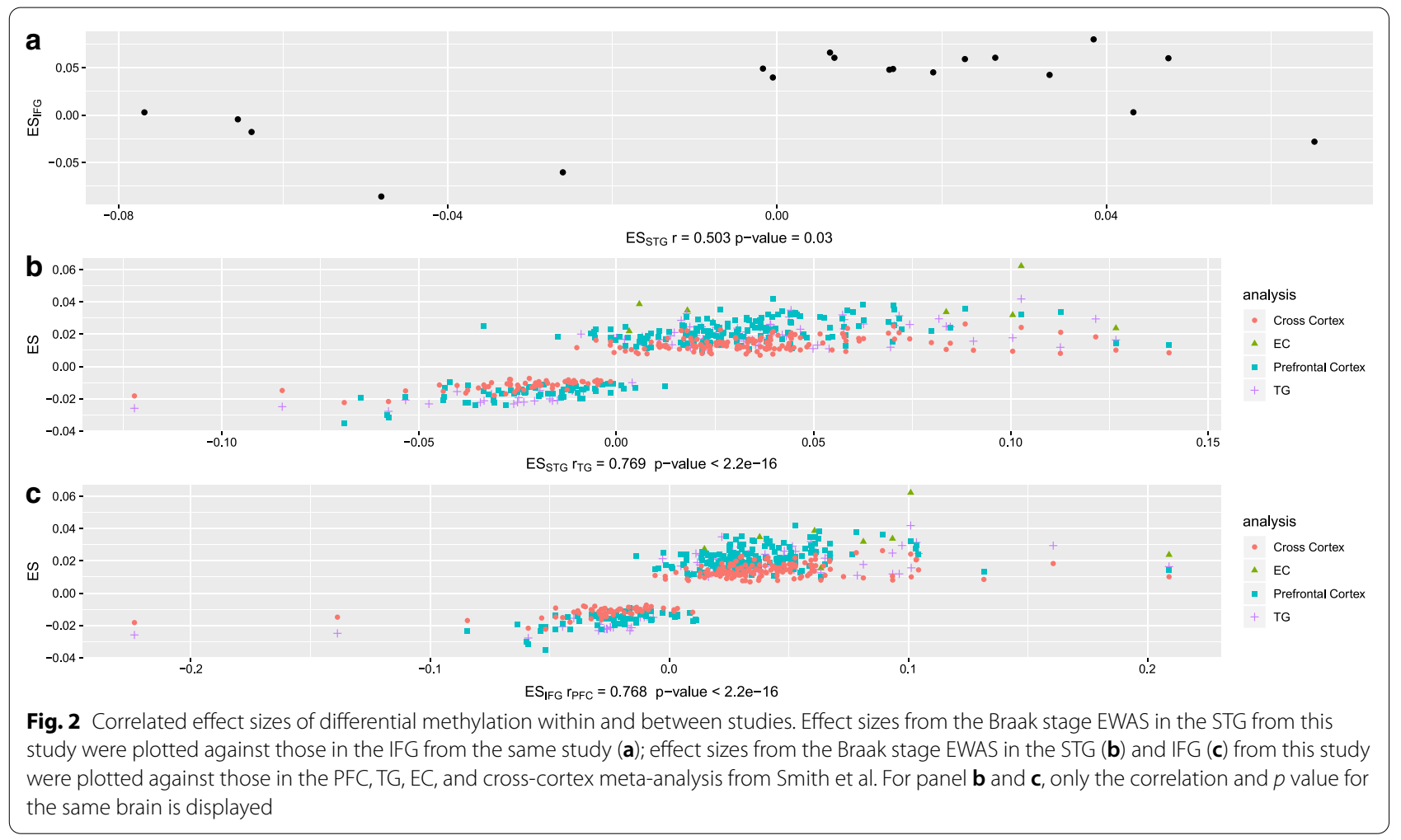

probes], Sidak-corrected $\left.p=9.65 \times 10^{-4}\right)$. The same gene cluster was identified in the STG with 3 DMRs spanning the HOXA3/HOXA-AS2/HOXA5 gene cluster [32 probes]. Majority of the probes in the $H O X A$ gene cluster were hypermethylated.

In addition, 4 DMRs associated with pathology in the IFG were detected in regions annotated to MCF2L consisting of a total of 17 probes (Additional file 1: Figure $\mathrm{S} 3 \mathrm{~B}$ ); the DMR annotated to $M C F 2 L$ was also detected in the STG. Furthermore, multiple DMRs annotated to TFAP2E (STG), ZNF608 (STG), STRA6 (IFG), LHX6 (IFG), SHH (IFG), and LINC00870 (IFG) were also detected. The $H O X A$ gene cluster, $M C F 2 L$, TFAP2E, SHH, and $L H X 6$, was among the replicated DMRs reported previously in the recent EWAS meta-analysis [21]. Additional replicated DMRs supported by a single DMR include regions annotated to $R G M A, C D 82, C P E B 4, R H B D F 2$ in both STG and IFG, C3, CUX2, CLDN5, CXXC5, DDAH2, DIP2A, PARS2, S1PR4, SLC16A3, HLA-DPA1, SMG9, ATP2A3, ZNF385A, DUSP27, CAMTA1, and the HOXB gene cluster (Additional file 1: Figure S3C) in the STG, and NAT8L, DDR1, SLC15A4, RHOB in the IFG (see Additional file 2: Tables S4A and S4B for a full list of replicated DMRs). In total, 26 (16.3\%, calculated at the gene level) of the reported 262 significant DMRs (annotated to 160 unique genes) from the PFC meta-analysis were replicated in the IFG analysis, while $15.8 \%$ of our 173 significant DMRs (annotated to 164 unique genes) were reciprocally replicated by the PFC meta-analysis. In addition, $11(18.3 \%)$ of the reported 104 significant DMRs (annotated to 60 unique genes) from the TG meta-analysis were replicated in the STG analysis, while $9.2 \%$ of our 121 significant DMRs (annotated to 119 unique genes) were reciprocally replicated by the TG meta-analysis. The replication rate at the DMR level seems to be higher than that at the DMP level.

Among the top DMRs associated with pathology in the IFG, the DMR annotated to DDAH2 stood out as one of the most significant DMRs (chr6:31,695,970-31,696,867 [26 probes], Sidak-corrected $\left.p=1.82 \times 10^{-13}\right)$. All of the 26 probes including a genome-wide significant DMP cg25845158 $\left(p=2.55 \times 10^{-8}\right.$ in the IFG) located in the CpG island were hypermethylated (Additional file 1: Figure S3D). A similar DMR (chr6:31,695,973-31,696,729 [21 probes], Sidak-corrected $p=1.37 \times 10^{-5}$ ) associated with pathology was also identified in the STG from this study and in the PFC in the recent EWAS meta-analysis (chr6:31,695,027-31,695,064 [3 probes], Sidak-corrected $\left.p=8.13 \times 10^{-4}\right)$. DDAH2 encodes dimethylarginine dimethylaminohydrolase 2 , an enzyme that functions in nitric oxide generation by regulating the cellular concentrations of methylarginines, which in turn inhibits nitric oxide synthase (NOS) activity. 


\section{AD-associated DMRs are enriched/depleted in specific genomic features}

Among the DMRs that were associated with pathology, genomic features such as promoter and CpG island (CGI) are highly enriched in all analyses. Exon, $5^{\prime}$ UTR, and transcription termination site (TTS) are also enriched. In contrast, intergenic region and repeats (SINE and LINE) are depleted (Fig. 3 and Additional file 2: Table S4).

\section{Gene set enrichment analysis (GSEA)}

\section{and over-representation analysis (ORA) results}

Gene set enrichment analysis of the DMPs and DMRs revealed that gene sets involved in neuroinflammation, neurogenesis, and cognition were enriched (false discovery rate $(F D R)<0.05$. See Additional file 2: Tables S5 and S6 for a full list of enriched gene sets from the DMP and DMR analyses).

Gene sets related to neuroinflammation were enriched in this study. Over representation analysis revealed that genes involved in microglia differentiation (driven by TSPAN2 and negative regulator of reactive oxygen species $(N R R O S)$ ) were enriched among the DMRs associated with pathology in the STG (Additional file 2: Table S5A). Noticeably, the DMR in NRROS was also replicated in the recent EWAS meta-analysis [21].
Phagocytosis $(p=0.001$, adjusted $p$ value $=0.05)$ was also enriched in DMPs associated with pathology in the IFG (Additional file 2: Table S5B).

For DMPs associated with pathology in the STG, positive regulation of neurogenesis $(p=0.001$, adjusted $p$ value $=0.09$ ) was also enriched (Additional file 2: Table S5A). Likewise, genes involved with neurogenesis were also enriched among the DMRs in the STG (Additional file 1: Table S6). Neurogenesis is critical for learning. Moreover, learning or memory $(p=0.001$, adjusted $p$ value $=0.05)$ was enriched among DMPs associated with pathology in the IFG (Additional file 1: Table S5B). Over-representation analysis for DMRs associated with pathology in the IFG suggested that genes involved in cognition were enriched (Additional file 2: Table S6), and this enrichment was driven by DMRs in zinc finger protein 385A (ZNF385A), CREB-regulated transcription coactivator 1 (CRTC1), SH3 and multiple ankyrin repeat domains 2 (SHANK2), cut-like homeobox 2 (CUX2), cytoplasmic FMR1 interacting protein 1 (CYFIP1), claudin 5 (CLDN5), stimulated by retinoic acid 6 (STRA6), and janus kinase and microtubule interacting protein 1 (JAKMIP1). Among these DMRs, ZNF385A, CUX2, and $C L D N 5$ were replicated in the recent EWAS meta-analysis [21].

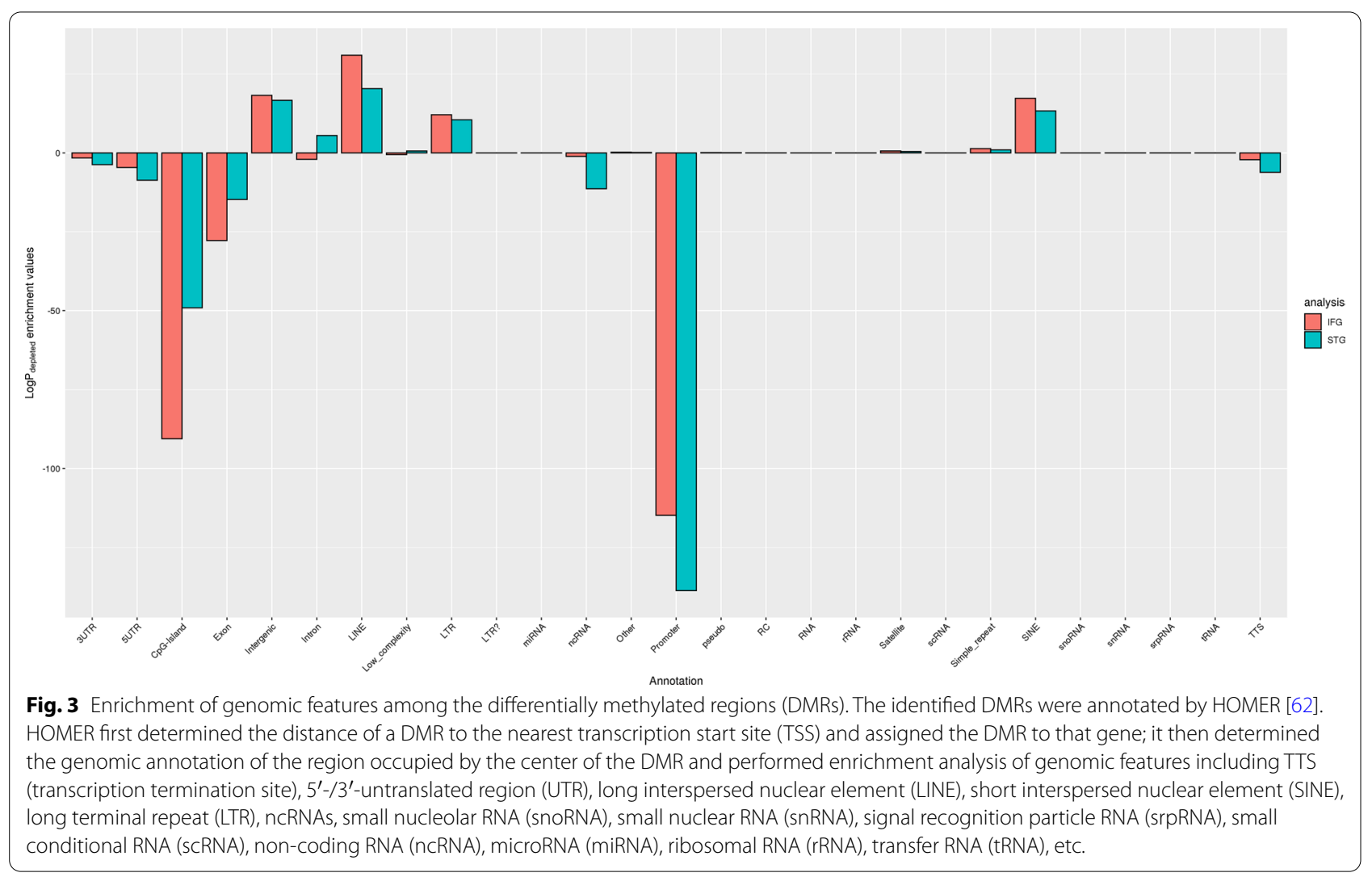




\section{Correlation between selected CpG probes} and between $\mathrm{CpG}$ probes and transcript level

Correlation among top ANK1 CpG cg13609385 (nominally associated with diagnosis in the STG, $p_{D M P}=0.001$ ), top $H O X A$ gene cluster probe cg14058329, top $H O X B$ gene cluster probe cg04904318 $\left(p_{D M P}=8.79 \times 10^{-6}\right)$, and top $D D A H 2$ probe $\mathrm{cg} 25845158$, and between these probes and expression level of all transcripts, were tested. Top HOXB gene cluster probe cg04904318 was correlated with multiple CpG probes. For example, cg13609385 was positively correlated $\left(r=0.74, p=1.33 \times 10^{-14}\right)$ with cg04904318 in the STG, but negatively correlated with cg04904318 $\left(r=-p\right.$ valu0.72, $p=2.06 \times 10^{-11}$, Additional file 1: Table S7) in the IFG, suggesting a brain region specificity in their interaction. This correlation is significant even after multiple testing correction $\left(p<0.05 /\left(4^{*} 2^{*}(5772+3)\right) \sim 1.08 \times 10^{-6}\right.$ to correct for testing correlation with 5,772 transcripts and 3 other CpG probes for a total of 4 probes tested in 2 brain regions). In addition, there was a positive correlation between the methylation level of $D D A H 2$ probe cg25845158 and that of cg04904318 in both the STG and IFG $(r=0.549$, $p=2.77 \times 10^{-7}$ in the STG; $r=0.588, p=3.33 \times 10^{-7}$ in the IFG, Additional file 1: Figures S4A and 4B).

\section{Discussion}

In this study, we profiled methylome from the STG and IFG brain regions and attempted to identify evidence of replication of the reported DMPs and DMRs from a recent EWAS meta-analysis [21]. We identified DMRs with replication evidence and replicated 22 significant DMPs reported in the recent EWAS meta-analysis. In addition, we discovered novel DMPs and DMRs associated with pathology surpassing the genome-wide significance threshold $\left(p<6.79 \times 10^{-8}\right.$ for DMPs, and Sidak-corrected $p$ value $<0.05$ for DMRs) for future follow-up studies.

It is of interest to note that despite the modest sample size, the replication rate for DMRs was substantial. The DMRs identified in this study were supported by more probes than that of previous studies [20,21], owing to the fact that the EPIC BeadChip used in this study doubles the CpG probe density compared to the previous studies that utilized the HumanMethylation450 BeadChip. This suggests that the higher $\mathrm{CpG}$ probe density could increase the power of DMR detection, given comparable study sample size. Indeed, for this study the replication rate at the DMR level is higher than that at the DMP level. $8.33 \%$ of the DMPs and $16.25 \%$ of the DMRs reported in the PFC meta-analysis were replicated in the IFG analysis, while $4.49 \%$ of the DMPs and $18.33 \%$ of the DMRs reported in the TG meta-analysis were replicated in the STG analysis. It appears that this study replicated more DMPs identified from the IFG region than the STG region. This could be due to the differential power of the meta-analysis in that the sample size in the PFC metaanalysis is larger than that in the TG meta-analysis (sample size $n=959$ for the PFC vs. $n=608$ for the TG), and hence, more (and perhaps also more reliable) DMPs were discovered ( $n=236$ for the PFC vs. $n=95$ for the TG).

The activation of $H O X$ genes during differentiation was enriched among the DMPs associated with pathology in the STG (Additional file 2: Table S5A). This is not surprising given the single genome-wide significant DMP in the $H O X A$ gene cluster and multiple significant DMRs in both $H O X A$ and $H O X B$ gene clusters were identified in this study. Both $H O X A$ and $H O X B$ differential methylation findings were reported previously in the AD brain [17-21, 32]. Additionally, HOXA differential methylation was reported in the blood from patients with Down syndrome [33] and $H O X B$ differential methylation was also reported in the blood from patients with AD [34]. Many Down syndrome patients develop AD resulting from an extra copy of the APP gene due to trisomy on chromosome 21. In Drosophila, it has been shown that the HOX transcription factor is one of the upstream regulators coordinating ankyrin-dependent microtubule organization and synapse stability [35] and it is therefore potentially neuroprotective. ANK1 was also reported to be differentially methylated in prior studies $[16-18,32]$ and replicated in this study. We therefore tested the correlation of top ANK1 CpG cg13609385 and representative probes in the $H O X$ gene clusters. Surprisingly, cg13609385 was positively correlated with the lead $H O X B$ probe cg04904318 in the STG, but negatively correlated with cg04904318 (Additional file 2: Table S7) in the IFG, suggesting a brain region specificity in their interaction.

Given the role of $D D A H 2$ in oxidative stress response, hypermethylation of $D D A H 2$ could theoretically result in lower level of $D D A H 2$ gene expression and increased NOS activity, excessive reactive oxygen species (ROS) production, and higher level of neuroinflammation, as shown previously that $D D A H 2$ expression level was inversely correlated with proinflammatory cytokines IL-6 and TNF-alpha [36]. However, with limited overlapping samples ( $n=76$ for the STG samples) between mRNA-Seq and EPIC array samples, we did not have evidence to support the negative correlation between the genome-wide significant DMP cg25845158 from $D D A H 2$ and $D D A H 2$ mRNA level, suggesting the transcriptional regulation of $D D A H 2$ is more complicated than simple regulation by the methylation at the $\mathrm{CpG}$ island. In contrast, there was a nominal positive correlation between cg25845158 and DDAH2 mRNA level in the IFG $(r=0.316, p=0.01$, Additional file 1: Figure S4C) 
and no correlation in the STG (Additional file 1: Fig. 4D). Interestingly, there was a positive correlation between the methylation level of $D D A H 2$ probe cg25845158 and the methylation level of the lead probe in the $H O X B$ gene cluster cg04904318 in both the STG and IFG. The significance of this correlation is unknown. Furthermore, $D D A H 2$ probe cg25845158 was negatively correlated with the transcript level of SNAP25, $(r=-0.62$, $\left.p=4.34 \times 10^{-8}\right)$, MAPK8IP2 $\left(r=-0.59, p=2.63 \times 10^{-}\right.$ 7), PDE $2 A\left(r=-0.59, p=2.86 \times 10^{-7}\right)$, and BZRAP1 $\left(r=-0.57, p=1.01 \times 10^{-6}\right)$ (Additional file 1: Table S7). This is of interest as it is thought that $A \beta$ peptides trigger synaptic dysfunction by interfering with the synaptic vesicular fusion facilitated by the SNARE protein complexes including SNAP25 [37]. MAPK8IP2 is also known as JIP2, c-Jun $\mathrm{NH}(2)$-terminal kinase (JNK)-interacting protein 2 , which is known to interact with $A \beta$ to play an important role in the metabolism and/or the function of $A \beta$ including the regulation of $A \beta$ phosphorylation by JNK [38]. Inhibition of PDE2 has been shown to rescue $\mathrm{A} \beta$ induced memory impairment via regulation of PKA/ PKG-dependent neuroinflammatory and apoptotic pathways [39]. Finally, genetic variants from BZRAP1-AS1 were previously implicated to be associated with $\mathrm{AD}$ [40].

While this study identified interesting genes and pathways, there are limitations that worth commenting. Despite the modest sample size for the two brain regions included in this study, the sample size is still far smaller compared to the pooled sample size in the recent metaanalysis, and hence limits the power in identifying more genome-wide significant DMPs and DMRs. This is a cross-sectional study using samples from the end stage of a disease and therefore it is difficult to infer whether the methylation change is causal or is a result of the disease process. The current study annotates $\mathrm{CpG}$ probes to the nearby genes based on the genomic location. It is possible that a regulatory element may interact with another sequence element in the distance via chromatin loop, and therefore, the functional consequence could affect another distal gene. This is a study using DNA extracted from bulk tissue despite the correction for neuronal proportion in the DMP analysis. Studies on cell-type specific methylation pattern $[19,32]$ revealed cell-type specific effect, which could be obscured in studies using bulk tissues. Finally, this study did not distinguish between $5-\mathrm{mC}$ and 5 -hmC and could have missed specific differences between the two. Further studies are needed to replicate the novel DMPs or DMRs identified in this study.

\section{Conclusions}

We conducted a modest size EWAS to identify DMPs and DMRs associated with pathology. Five and 14 study-wide significant DMPs were identified to be associated with pathology in the STG and IFG, respectively. Our study replicated 22 DMPs supporting the findings of a recent EWAS meta-analysis. Additionally, there was substantial overlap between the DMRs identified in this study and those identified in the recent meta-analysis. The identified DMPs and DMRs converged on biological pathways and processes that were previously implicated in AD.

\section{Methods \\ Cohort}

Postmortem brain case samples from patients with $\mathrm{AD}$ and control samples from patients who were cognitively normal from the STG $\left(n_{\text {case }}=91, n_{\text {control }}=61\right)$ and IFG $\left(n_{\text {case }}=89, n_{\text {control }}=57\right)$ were acquired from Banner Sun Health Research Institute [41, 42]. These brain samples came from subjects who were volunteers in the Arizona Study of Aging and Neurodegenerative Disorders (AZSAND) and the Brain and Body Donation Program, a longitudinal clinicopathological study of healthy aging, cognition, and movement in the elderly since 1996 in Sun City, Arizona.

\section{Postmortem brain tissue epigenetic profiling}

Genomic DNA and total RNA, including miRNA, were simultaneous purified from the brain tissue samples using AllPrep DNA/RNA/miRNA Universal Kit (QIAGEN Inc., Germantown, MD, USA) following the standard protocol. $10 \mu \mathrm{l}$ of genomic DNA with minimal concentration of $40 \mathrm{ng} / \mu \mathrm{l}$ was bisulfite converted using the Zymo EZ DNA Methylation ${ }^{\mathrm{TM}}$ kit (Zymo, Irvine, CA, USA) using the manual protocol, while genome-wide methylation was measured using Infinium ${ }^{\circledR}$ MethylationEPIC BeadChip (Illumina, San Diego, CA, USA) using the automated protocol as detailed in the Infinium ${ }^{\circledR} \mathrm{HD}$ Assay Methylation Protocol. Methylome profiling data were generated over two batches for each brain region, respectively. All data generation were conducted by laboratory personnel blinded as to the clinical phenotype.

\section{Postmortem brain tissue mRNA-Seq}

The mRNA-Seq study was reported previously [43]. RNA samples $\left(n_{\text {case }}=24, n_{\text {control }}=38\right)$ from the same cohort above with RNA integrity number (RIN) greater than 6 were proceeded to the library construction step for mRNA-Seq data generation. Libraries were constructed using TruSeq ${ }^{\circledR}$ Stranded mRNA Library Prep (Illumina Inc., San Diego, CA, USA) according to manufacturer's protocol using $200 \mathrm{ng}$ of input RNA. Briefly, poly-Acontaining mRNA was captured using poly-T oligonucleotide-attached magnetic beads. Following purification, the mRNA was fragmented using divalent cations under elevated temperature. The cleaved RNA fragments were copied into first strand cDNA using reverse transcriptase 
and random primers. Strand specificity was achieved by replacing dTTP with dUTP in the Second Strand Marking Mix (SMM), followed by second strand cDNA synthesis using DNA polymerase I and RNase $\mathrm{H}$. These cDNA fragments were then followed by A-tailing and adapter ligation reactions. The products were purified and enriched with PCR to create the final cDNA library. All libraries were quantified by Caliper and real-time qPCR and amplified on cBot to generate the clusters on the flowcell, and sequenced using HiSeq4000 (Illumina Inc., San Diego, CA, USA) using paired end $(100 \mathrm{bp} \times 2)$ sequencing to a sequencing depth of $40 \mathrm{M}$ reads (or $8 \mathrm{G}$ data). Sequencing data were generated over two batches for each brain region. All data generation were conducted by laboratory personnel blinded as to the clinical phenotype. This dataset was used to perform correlation analysis of selected $\mathrm{CpG}$ probes and mRNA transcript to shed light on the potential consequence of DNA methylation.

\section{Data Pre-processing}

Epigenetic data were analyzed separately for each brain region/wave. Quality control of the epigenetic data was performed using ChAMP R package [44]. Probes that did not perform well (with detection $p$ value $\geq 0.01$ in one or more samples $\left(n_{\mathrm{STG}}=10,136\right.$ and $n_{\mathrm{IFG}}=10,920$ for the STG and IFG samples, respectively), or with bead count $<3$ in at least $5 \%$ of samples $\left(n_{\mathrm{STG}}=4220\right.$ and $n_{\mathrm{IFG}}=7179$ ), probes with known SNP sites or with cross-reactivity [45] $\left(n_{\mathrm{STG}}=95,414\right.$ and $\left.n_{\mathrm{IFG}}=94,915\right)$, non-CG probes $\left(\mathrm{n}_{\mathrm{STG}}=2910\right.$ and $\left.\mathrm{n}_{\mathrm{IFG}}=2900\right)$, probes align to multiple locations on the genome [46] $(n=15)$, as well as probes located on the sex chromosomes $\left(n_{\mathrm{STG}}=16,400\right.$ and $\left.n_{\mathrm{IFG}}=16,287\right)$ were filtered out. At the sample level, gender based on the methylation data was estimated using getSex function in the minfi (v1.28.4) $\mathrm{R}$ package and compared to that from the clinical phenotype. No discrepant gender was detected for the study samples. Since a subject may have samples from two brain regions assayed in this study, sample identity check was performed using R package ewastools (v1.6) [47]. All expected pairs of identity were confirmed, and all detected pairs of identity were expected.

The methylation levels were then normalized using Dasen method in R package wateRmelon [48]. The neuronal vs. non-neuronal cell composition was estimated using the estimateCellCounts function in minfi [49] which used a reference brain dataset of fluorescence activated cell sorting (FACS) sorted neuronal and nonneuronal nuclear fractions [50]. Surrogate variables are covariates constructed directly from high-dimensional data that could be used in subsequent analyses to adjust for unknown, unmodeled, or latent sources of noise [51, 52]. We used sva (v3.30.1) $[53,54]$ to detect and estimate surrogate variables for unknown sources of variation to remove artifacts in the high-throughput experiments. Removing batch effects using surrogate variables in differential analysis have been shown to reduce dependence, stabilize error rate estimates, and improve reproducibility [55]. Samples with discrepant phenotype between sample label and the phenotype data linked to case ID on the sample label were excluded from downstream analysis. To have a balanced age-matched study design, only samples from subjects aged between 60 and 89 inclusive were included in the analysis resulting in sample sizes of 127 samples (67 AD and 60 cognitively normal control) and 117 samples (60 AD and 57 cognitively normal control) for the STG and IFG, respectively, used in downstream analysis.

mRNA-Seq data were processed per sample using cutadapt (v1.13) [56], and STAR (v2.5.3a) [57]. Transcript quantification was performed using RSEM (v1.3.0) [58] against all 26,000 genes in NCBI RefSeq database (version date; 2015-07-17).

\section{Identification of DMPs}

We used $M$ value in the statistical analysis to identify DMPs using limma [59] as M value was shown to provide better performance in detection rate and true positive rate for both highly methylated and unmethylated CPG sites and was more statistically valid than betavalue, despite beta-value was more biologically intuitive [60]. Epigenetic association model corrected the top five surrogate variables, sex, age, neuronal proportion, and Braak stage (as a continuous variable) was tested in a linear regression model to identify differentially methylated probes associated with Braak stage. Epigenome-wide association studies were prone to significant inflation and bias of test statistics, and a Bayesian method to control bias and inflation in EWAS based on estimation of the empirical null distribution was proposed and implemented in R package BACON [30]. We applied this Bayesian method as implemented in BACON v1.10.1 to control for inflation and lambda (l) inflation factors before and after correction was reported. A stringent threshold using Bonferroni correction was used to declare study-wide significance.

The discovered DMPs in each brain region were examined for consistency evidence in several ways. Firstly, we checked for consistency of effect size and directionality between the two brain regions in this study; secondly, we compared the effect sizes from this study to those reported in the recent meta-analysis [21]. Lastly, we attempted to replicate the published genome-wide significant DMPs and DMRs from the recent meta-analysis given that our data were generated using Illumina EPIC 
BeadChip, and the published studies were using the Illumina HumanMethylation450 BeadChip.

\section{Identification and annotation of DMRs and genomic feature enrichment}

DMRs were identified using comb-p [61] with a distance of $500 \mathrm{bp}$ and a seeded $p$ value of $1.0 \times 10^{-4}$. The DMR analyses were carried out for all probes (irrespective of directionality of differential methylation), and DMRs with at least three probes and Sidak-corrected $p$ less than 0.05 were considered significant and reported. The identified DMRs were annotated by HOMER [62]. HOMER first determined the distance of a DMR to the nearest transcription start site (TSS) and assigned the DMR to that gene, it then determined the genomic annotation of the region occupied by the center of the DMR and performed enrichment analysis of genomic features.

\section{Gene set enrichment analysis}

ORA [63] for genes near significant CpGs from Illumina's Infinium Human MethylationEPIC array was performed using missMethyl $\mathrm{R}$ package v1.16.0 [64], taking into account the differing number of probes per gene present on the array. Additionally, a GSEA [65] analysis was performed using $\mathrm{R}$ package methylGSA [66] adjusting for multiple $p$ values of each gene by Robust Rank Aggregation (RRA), and then apply pre-ranked version of GSEA (GSEAPreranked) in gene set testing. Lastly, methylglm function within $\mathrm{R}$ package methylGSA was used for length bias correction using logistic regression [67].Gene ontology databases used included KEGG database [68] and c2.cp (a superset of c2.cp.biocarta, c2.cp.kegg, and c2.cp.reactome [69] and a few other data sources) (v7.0) subsets of Molecular signatures database (MSigDB) [70].

Over-representation analysis of genes implicated by DMR was performed using https://www.gsea-msigd b.org/gsea/msigdb/compute_overlaps.jsp which has a broader background gene set assumption and test overrepresentation at higher levels of the ontology hierarchy. Gene ontology databases used included c2.cp and c5 subsets of MSigDB [70].

\section{Methylation-mRNA correlation analysis}

In order to identify potential consequence of DNA methylation, paired methylation level-mRNA correlation analysis was performed using Partek Genomic Suite (Partek Inc, St Louis, MO, USA), which only examined the correlation between selected top CpG probes $(n=4)$ and the transcript level. We used fsva function in sva $R$ package to perform frozen surrogate variable analysis [71] to remove nuisance batch effects from both methylation array and mRNA-Seq datasets and used the adjusted version of datasets for correlation analysis. Multiple testing correction was applied $\left(p<0.05 /\left(4^{*} 2^{*}(5772+3)\right) \sim 1.08 \times 10^{-6}\right.$ to correlate for testing correlation with 5772 transcripts (only test the moderate to abundant transcripts) and 3 other $\mathrm{CpG}$ probes for 4 probes in 2 brain regions) as some of top CpG probes are in the homeobox transcription factors, and the functional consequence could be reflected in the targets of the transcriptional factors.

\section{Supplementary information}

Supplementary information accompanies this paper at https://doi. org/10.1186/s13148-020-00944-z.

Additional file 1. Supplemental Figures S1-S4.

Additional file 2. Supplemental Tables S1-S7.

\section{Abbreviations}

AD: Alzheimer's disease; EWAS: Epigenome-wide association study; STG: Superior temporal gyrus; IFG: Inferior frontal gyrus; EC: Entorhinal cortex; FDR: False discovery rate; DMP: Differentially methylated position; DMR: Differentially methylated region; GWAS: Genome-wide association studies; NGS: Next generation sequencing; 5-mC: 5-Methylcytosine; 5-hmC: 5-Hydroxymethylcytosine; NFT: Neurofibrillary tangle; DEG: Differentially expressed gene; GEO: Gene expression omnibus; GSEA: Gene set enrichment analysis; ORA: Over-representation analysis; RIN: RNA integrity number; FACS: Fluorescence activated cell sorting; RRA: Robust rank aggregation; MSigDB: Molecular signatures database.

\section{Acknowledgements}

We thank the patients for participating in the brain donor program and the researchers at Banner Sun Research Institutes for making the samples available to research communities. We also thank the staff at Cancer Genetics, Inc. for extracting nucleic acids from the samples, quality control, and the staff at Cancer Genetics, Inc. and HD Bio for plating the samples for assay, the staff at Illumina for generating the epigenetic assay data, and the staff at BGI for generating the mRNA-Seq assay data. The epigenetic and mRNA-Seq data from postmortem samples reported in this study were generated from samples collected through the Sun Health Research Institute Brain and Body Donation Program of Sun City, Arizona. The Brain and Body Donation Program is supported by the National Institute of Neurological Disorders and Stroke (U24 NS072026 National Brain and Tissue Resource for Parkinson's Disease and Related Disorders), the National Institute on Aging (P30 AG19610 Arizona Alzheimer's Disease Core Center), the Arizona Department of Health Services (Contract 211002, Arizona Alzheimer's Research Center), the Arizona Biomedical Research Commission (Contracts 4001, 0011, 05-901, and 1001 to the Arizona Parkinson's Disease Consortium), and the Michael J. Fox Foundation for Parkinson's Research.

\section{Authors' contributions}

QSL conceived the project and designed the study. QSL, YS, and TW undertook the data analysis and bioinformatics. QSL drafted the manuscript. All authors provided feedback and approved the final submission. All authors read and approved the final manuscript.

Funding

The study was funded by Janssen Research \& Development, LLC.

\section{Availability of data and materials}

The EPIC array dataset generated and analyzed during the current study is available from GEO under the accession number GSE156984. The code snippets associated with this manuscript are available at https://github.com/qsere nali/EWAS. 


\section{Ethics approval and consent to participate}

All subjects signed an Institutional Review Board-approved informed consent, allowing both clinical assessments during life and several options for brain and bodily organ donation after death.

\section{Consent for publication}

Not applicable.

\section{Competing interests}

QSL and YS are employees of Janssen Research \& Development, LLC and may own stock/stock options in the company. TW was employed by AccuraScience, LLC.

\section{Author details}

${ }^{1}$ Neuroscience, Janssen Research \& Development, LLC, 1125 Trenton-Harbourton Road, Titusville, NJ 08560, USA. ${ }^{2}$ AccuraScience, LLC, Johnston, IA USA. ${ }^{3}$ Present Address: Discovery Science, Janssen Research \& Development, LLC, Spring House, PA, USA. ${ }^{4}$ Present Address: Center for Medical Genetics and Hunan Key Laboratory of Medical Genetics, School of Life Sciences, Central South University, Changsha 410083, China. ${ }^{5}$ Present Address: Beijing Institutes of Life Science, Chinese Academy of Sciences, Beijing 100101, China.

Received: 2 June 2020 Accepted: 7 October 2020

Published online: 17 October 2020

\section{References}

1. Lane CA, Hardy J, Schott JM. Alzheimer's disease. Eur J Neurol. 2018;25:59-70.

2. Cacace R, Sleegers K, Van Broeckhoven C. Molecular genetics of earlyonset Alzheimer's disease revisited. Alzheimer's Dementia J Alzheimer's Assoc. 2016;12:733-48.

3. Kunkle BW, Grenier-Boley B, Sims R, Bis JC, Damotte V, Naj AC, Boland A, Vronskaya M, van der Lee SJ, Amlie-Wolf A, et al. Genetic meta-analysis of diagnosed Alzheimer's disease identifies new risk loci and implicates Abeta, tau, immunity and lipid processing. Nat Genet. 2019;51:414-30.

4. Liu JZ, Erlich Y, Pickrell JK. Case-control association mapping by proxy using family history of disease. Nat Genet. 2017;49:325-31.

5. Lambert JC, Ibrahim-Verbaas CA, Harold D, Naj AC, Sims R, Bellenguez C, DeStafano AL, Bis JC, Beecham GW, Grenier-Boley B, et al. Meta-analysis of 74,046 individuals identifies 11 new susceptibility loci for Alzheimer's disease. Nat Genet. 2013;45:1452-8.

6. Jansen IE, Savage JE, Watanabe K, Bryois J, Williams DM, Steinberg S, Sealock J, Karlsson IK, Hagg S, Athanasiu L, et al. Genome-wide meta-analysis identifies new loci and functional pathways influencing Alzheimer's disease risk. Nat Genet. 2019;51:404-13.

7. Marioni RE, Harris SE, Zhang Q, McRae AF, Hagenaars SP, Hill WD, Davies G, Ritchie CW, Gale CR, Starr JM, et al. GWAS on family history of Alzheimer's disease. Transl Psychiatry. 2018;8:99.

8. Farrer LA, Cupples LA, Haines JL, Hyman B, Kukull WA, Mayeux R, Myers $\mathrm{RH}$, Pericak-Vance MA, Risch N, van Duijn CM. Effects of age, sex, and ethnicity on the association between apolipoprotein $\mathrm{E}$ genotype and Alzheimer disease. A meta-analysis. APOE and Alzheimer Disease Meta Analysis Consortium. JAMA. 1997;278:1349-56.

9. Cuyvers E, Sleegers K. Genetic variations underlying Alzheimer's disease: evidence from genome-wide association studies and beyond. Lancet Neurol. 2016;15:857-68.

10. Guerreiro R, Wojtas A, Bras J, Carrasquillo M, Rogaeva E, Majounie E, Cruchaga C, Sassi C, Kauwe JS, Younkin S, et al. TREM2 variants in Alzheimer's disease. N Engl J Med. 2013;368:117-27.

11. Jonsson T, Stefansson H, Steinberg S, Jonsdottir I, Jonsson PV, Snaedal J, Bjornsson S, Huttenlocher J, Levey Al, Lah JJ, et al. Variant of TREM2 associated with the risk of Alzheimer's disease. N Engl J Med. 2013:368:107-16.

12. Smith RG, Lunnon K. DNA modifications and Alzheimer's disease. Adv Exp Med Biol. 2017;978:303-19.

13. Lahiri DK, Maloney B, Zawia NH. The LEARn model: an epigenetic explanation for idiopathic neurobiological diseases. Mol Psychiatry. 2009;14:992-1003.
14. Maloney B, Lahiri DK. Epigenetics of dementia: understanding the disease as a transformation rather than a state. Lancet Neurol. 2016;15:760-74.

15. Maloney B, Sambamurti K, Zawia N, Lahiri DK. Applying epigenetics to Alzheimer's disease via the latent early-life associated regulation (LEARn) model. Curr Alzheimer Res. 2012;9:589-99.

16. Smith AR, Smith RG, Pishva E, Hannon E, Roubroeks JAY, Burrage J, Troakes C, Al-Sarraj S, Sloan C, Mill J, et al. Parallel profiling of DNA methylation and hydroxymethylation highlights neuropathology-associated epigenetic variation in Alzheimer's disease. Clin Epigenet. 2019;11:52.

17. De Jager PL, Srivastava G, Lunnon K, Burgess J, Schalkwyk LC, Yu L, Eaton ML, Keenan BT, Ernst J, McCabe C, et al. Alzheimer's disease: early alterations in brain DNA methylation at ANK1, BIN1, RHBDF2 and other loci. Nat Neurosci. 2014;17:1156-63.

18. Lunnon K, Smith R, Hannon E, De Jager PL, Srivastava G, Volta M, Troakes C, Al-Sarraj S, Burrage J, Macdonald R, et al. Methylomic profiling implicates cortical deregulation of ANK1 in Alzheimer's disease. Nat Neurosci. 2014;17:1164-70.

19. Hernandez HG, Sandoval-Hernandez AG, Garrido-Gil P, Labandeira-Garcia JL, Zelaya MV, Bayon GF, Fernandez AF, Fraga MF, Arboleda G, Arboleda H. Alzheimer's disease DNA methylome of pyramidal layers in frontal cortex: laser-assisted microdissection study. Epigenomics. 2018;10:1365-82.

20. Smith RG, Hannon E, De Jager PL, Chibnik L, Lott SJ, Condliffe D, Smith AR, Haroutunian V, Troakes C, Al-Sarraj S, et al. Elevated DNA methylation across a 48-kb region spanning the HOXA gene cluster is associated with Alzheimer's disease neuropathology. Alzheimer's Dementia J Alzheimer's Assoc. 2018. https://doi.org/10.1016/j.jalz.2018.01.017.

21. Smith RG, Pishva E, Shireby G, Smith AR, Roubroeks JAY, Hannon E, Wheildon G, Mastroeni D, Gasparoni G, Riemenschneider M, et al. Meta-analysis of epigenome-wide association studies in Alzheimer's disease highlights 220 differentially methylated loci across cortex. bioRxiv. 2020. https://doi. org/10.1101/2020.02.28.957894v1.

22. Smith AR, Smith RG, Condliffe D, Hannon E, Schalkwyk L, Mill J, Lunnon $\mathrm{K}$. Increased DNA methylation near TREM2 is consistently seen in the superior temporal gyrus in Alzheimer's disease brain. Neurobiol Aging. 2016:47:35-40

23. Halliday GM, Double KL, Macdonald V, Kril JJ. Identifying severely atrophic cortical subregions in Alzheimer's disease. Neurobiol Aging. 2003:24:797-806.

24. Youssef P, Chami B, Lim J, Middleton T, Sutherland GT, Witting PK. Evidence supporting oxidative stress in a moderately affected area of the brain in Alzheimer's disease. Sci Rep. 2018;8:11553.

25. Mills JD, Sheahan PJ, Lai D, Kril JJ, Janitz M, Sutherland GT. The alternative splicing of the apolipoprotein $\mathrm{E}$ gene is unperturbed in the brains of Alzheimer's disease patients. Mol Biol Rep. 2014;41:6365-76.

26. Jernigan TL, Archibald SL, Fennema-Notestine C, Gamst AC, Stout JC, Bonner J, Hesselink JR. Effects of age on tissues and regions of the cerebrum and cerebellum. Neurobiol Aging. 2001;22:581-94.

27. Resnick SM, Pham DL, Kraut MA, Zonderman AB, Davatzikos C. Longitudinal magnetic resonance imaging studies of older adults: a shrinking brain. J Neurosci. 2003;23:3295-301.

28. Sowell ER, Peterson BS, Thompson PM, Welcome SE, Henkenius AL, Toga AW. Mapping cortical change across the human life span. Nat Neurosci. 2003:6:309-15

29. Watson CT, Roussos P, Garg P, Ho DJ, Azam N, Katsel PL, Haroutunian $\checkmark$, Sharp AJ. Genome-wide DNA methylation profiling in the superior temporal gyrus reveals epigenetic signatures associated with Alzheimer's disease. Genome Med. 2016:8:5

30. van Iterson M, van Zwet EW, Consortium B, Heijmans BT. Controlling bias and inflation in epigenome- and transcriptome-wide association studies using the empirical null distribution. Genome Biol. 2017;18:19.

31. Yu L, Chibnik LB, Srivastava GP, Pochet N, Yang J, Xu J, Kozubek J, Obholzer $\mathrm{N}$, Leurgans SE, Schneider JA, et al. Association of Brain DNA methylation in SORL1, ABCA7, HLA-DRB5, SLC24A4, and BIN1 with pathological diagnosis of Alzheimer disease. JAMA Neurol. 2015;72:15-24.

32. Gasparoni G, Bultmann S, Lutsik P, Kraus TFJ, Sordon S, Vlcek J, Dietinger V, Steinmaurer $\mathrm{M}$, Haider M, Mulholland CB, et al. DNA methylation analysis on purified neurons and glia dissects age and Alzheimer's disease-specific changes in the human cortex. Epigenet Chromatin. 2018;11:41.

33. Bacalini MG, Gentilini D, Boattini A, Giampieri E, Pirazzini C, Giuliani C, Fontanesi E, Scurti M, Remondini D, Capri M, et al. Identification of a DNA 
methylation signature in blood cells from persons with Down Syndrome. Aging (Albany NY). 2015;7:82-96.

34. Roubroeks JAY, Smith AR, Smith RG, Pishva E, Ibrahim Z, Sattlecker M, Hannon EJ, Kłoszewska I, Mecocci P, Soininen H, et al. An epigenomewide association study of Alzheimer's disease blood highlights robustDNA hypermethylation in the HOXB6 gene. Neurobiol Aging. 2020;95:26-45.

35. Friedrich J, Sorge S, Bujupi F, Eichenlaub MP, Schulz NG, Wittbrodt J, Lohmann I. Hox function is required for the development and maintenance of the drosophila feeding motor unit. Cell Rep. 2016;14:850-60.

36. Puchau B, Hermsdorff HH, Zulet MA, Martinez JA. DDAH2 mRNA expression is inversely associated with some cardiovascular risk-related features in healthy young adults. Dis Markers. 2009;27:37-44.

37. Sharda N, Pengo T, Wang Z, Kandimalla KK. Amyloid-beta peptides disrupt interactions between VAMP-2 and SNAP-25 in neuronal cells as determined by FRET/FLIM. J Alzheimer's Dis JAD. 2020;77(1):423-35.

38. Taru H, lijima K, Hase M, Kirino Y, Yagi Y, Suzuki T. Interaction of Alzheimer's beta -amyloid precursor family proteins with scaffold proteins of the JNK signaling cascade. J Biol Chem. 2002;277:20070-8.

39. Wang L, Xiaokaiti Y, Wang G, Xu X, Chen L, Huang X, Liu L, Pan J, Hu S, Chen $Z$, Xu Y. Inhibition of PDE2 reverses beta amyloid induced memory impairment through regulation of PKA/PKG-dependent neuro-inflammatory and apoptotic pathways. Sci Rep. 2017;7:12044.

40. Witoelar A, Rongve A, Almdahl IS, Ulstein ID, Engvig A, White LR, Selbaek G, Stordal E, Andersen F, Braekhus A, et al. Meta-analysis of Alzheimer's disease on 9,751 samples from Norway and IGAP study identifies four risk loci. Sci Rep. 2018;8:18088.

41. Beach TG, Sue LI, Walker DG, Roher AE, Lue L, Vedders L, Connor DJ, Sabbagh MN, Rogers J. The Sun Health Research Institute Brain Donation Program: description and experience, 1987-2007. Cell Tissue Bank. 2008:9:229-45.

42. Beach TG, Adler CH, Sue LI, Serrano G, Shill HA, Walker DG, Lue L, Roher $A E$, Dugger BN, Maarouf C, et al. Arizona study of aging and neurodegenerative disorders and brain and body donation program. Neuropathology. 2015;35:354-89.

43. Li Q. M148 A mRNA-Seq study using post-mortem brain tissue samples in patients with Alzheimer's disease compared to cognitively normal control subjects. ACNP 58(th) annual meeting: poster session I. Neuropsychopharmacology. 2019;44:156.

44. Morris TJ, Butcher LM, Feber A, Teschendorff AE, Chakravarthy AR, Wojdacz TK, Beck S. ChAMP: 450k chip analysis methylation pipeline. Bioinformatics. 2014;30:428-30.

45. Zhou W, Laird PW, Shen H. Comprehensive characterization, annotation and innovative use of Infinium DNA methylation BeadChip probes. Nucleic Acids Res. 2017;45:e22.

46. Nordlund J, Backlin CL, Wahlberg P, Busche S, Berglund EC, Eloranta ML, Flaegstad T, Forestier E, Frost BM, Harila-Saari A, et al. Genome-wide signatures of differential DNA methylation in pediatric acute lymphoblastic leukemia. Genome Biol. 2013;14:r105.

47. Just AC, Heiss JA: ewastools: EWAS Tools. . R package version 162018.

48. Pidsley R, Wong CCY, Volta M, Lunnon K, Mill J, Schalkwyk LC. A datadriven approach to preprocessing Illumina $450 \mathrm{~K}$ methylation array data. BMC Genom. 2013;14:293.

49. Aryee MJ, Jaffe AE, Corrada-Bravo H, Ladd-Acosta C, Feinberg AP, Hansen KD, Irizarry RA. Minfi: a flexible and comprehensive bioconductor package for the analysis of infinium DNA methylation microarrays. Bioinformatics. 2014:30:1363-9.

50. Guintivano J, Aryee MJ, Kaminsky ZA. A cell epigenotype specific model for the correction of brain cellular heterogeneity bias and its application to age, brain region and major depression. Epigenetics. 2013;8:290-302.

51. Leek JT, Storey JD. Capturing heterogeneity in gene expression studies by surrogate variable analysis. PLoS Genet. 2007;3:1724-35.

52. Leek JT, Storey JD. A general framework for multiple testing dependence. Proc Natl Acad Sci USA. 2008:105:18718-23.

53. Leek JT, Johnson WE, Parker HS, Jaffe AE, Storey JD. The sva package for removing batch effects and other unwanted variation in high-throughput experiments. Bioinformatics. 2012;28:882-3.

54. Leek JT, Johnson WE, Parker HS, Fertig EJ, Jaffe AE, Storey JD, Zhang Y, Torres LC: sva: Surrogate Variable Analysis. R package version 3.30.1. 2019.
55. Leek JT, Scharpf RB, Bravo HC, Simcha D, Langmead B, Johnson WE, Geman D, Baggerly K, Irizarry RA. Tackling the widespread and critical impact of batch effects in high-throughput data. Nat Rev Genet. 2010;11:733-9.

56. Martin M. Cutadapt removes adapter sequences from high-throughput sequencing reads. EMBnetJournal. 2011;17:10-2.

57. Dobin A, Davis CA, Schlesinger F, Drenkow J, Zaleski C, Jha S, Batut $P$, Chaisson M, Gingeras TR. STAR: ultrafast universal RNA-seq aligner. Bioinformatics. 2013;29:15-21.

58. Li B, Dewey CN. RSEM: accurate transcript quantification from RNA-Seq data with or without a reference genome. BMC Bioinform. 2011;12:323.

59. Ritchie ME, Phipson B, Wu D, Hu Y, Law CW, Shi W, Smyth GK. limma powers differential expression analyses for RNA-sequencing and microarray studies. Nucleic Acids Res. 2015;43:e47.

60. Du P, Zhang X, Huang CC, Jafari N, Kibbe WA, Hou L, Lin SM. Comparison of Beta-value and $\mathrm{M}$-value methods for quantifying methylation levels by microarray analysis. BMC Bioinform. 2010;11:587.

61. Pedersen BS, Schwartz DA, Yang IV, Kechris KJ. Comb-p: software for combining, analyzing, grouping and correcting spatially correlated P-values. Bioinformatics. 2012;28:2986-8.

62. Heinz S, Benner C, Spann N, Bertolino E, Lin YC, Laslo P, Cheng JX, Murre C, Singh H, Glass CK. Simple combinations of lineage-determining transcription factors prime cis-regulatory elements required for macrophage and B cell identities. Mol Cell. 2010;38:576-89.

63. Boyle El, Weng S, Gollub J, Jin H, Botstein D, Cherry JM, Sherlock G. GO::TermFinder-open source software for accessing Gene Ontology information and finding significantly enriched Gene Ontology terms associated with a list of genes. Bioinformatics. 2004;20:3710-5.

64. Phipson B, Maksimovic J, Oshlack A. missMethyl: an R package for analyzing data from Illumina's HumanMethylation450 platform. Bioinformatics. 2016;32:286-8.

65. Subramanian A, Tamayo P, Mootha VK, Mukherjee S, Ebert BL, Gillette MA, Paulovich A, Pomeroy SL, Golub TR, Lander ES, Mesirov JP. Gene set enrichment analysis: a knowledge-based approach for interpreting genome-wide expression profiles. Proc Natl Acad Sci USA. 2005; 102:15545-50.

66. Ren X, Kuan PF. methylGSA: a Bioconductor package and Shiny app for DNA methylation data length bias adjustment in gene set testing. Bioinformatics. 2019;35:1958-9.

67. Mi G, Di Y, Emerson S, Cumbie JS, Chang JH. Length bias correction in gene ontology enrichment analysis using logistic regression. PLOS ONE. 2012; 7:e46128

68. Kanehisa M, Furumichi M, Tanabe M, Sato Y, Morishima K. KEGG: new perspectives on genomes, pathways, diseases and drugs. Nucleic Acids Res. 2017;45:D353-61.

69. Fabregat A, Jupe S, Matthews L, Sidiropoulos K, Gillespie M, Garapati P, Haw R, Jassal B, Korninger F, May B, et al. The reactome pathway knowledgebase. Nucleic Acids Res. 2018;46:D649-55.

70. Liberzon A, Subramanian A, Pinchback R, Thorvaldsdottir H, Tamayo P, Mesirov JP. Molecular signatures database (MSigDB) 3.0. Bioinformatics. 2011:27:1739-40.

71. Parker HS, Corrada Bravo H, Leek JT. Removing batch effects for prediction problems with frozen surrogate variable analysis. PeerJ. 2014;2:e561.

72. Hyman BT, Trojanowski JQ. Consensus recommendations for the postmortem diagnosis of Alzheimer disease from the National Institute on Aging and the Reagan Institute Working Group on diagnostic criteria for the neuropathological assessment of Alzheimer disease. J Neuropathol Exp Neurol. 1997;56:1095-7.

73. Mirra SS, Heyman A, McKeel D, Sumi SM, Crain BJ, Brownlee LM, Vogel FS, Hughes JP, van Belle G, Berg L. The Consortium to Establish a Registry for Alzheimer's Disease (CERAD). Part II. Standardization of the neuropathologic assessment of Alzheimer's disease. Neurology. 1991;41:479-86.

\section{Publisher's Note}

Springer Nature remains neutral with regard to jurisdictional claims in published maps and institutional affiliations. 